Kardiologe 2021 · 15:585-594

https://doi.org/10.1007/s12181-021-00505-5

Angenommen: 4. August 2021

Online publiziert: 4. Oktober 2021

(c) Deutsche Gesellschaft für Kardiologie - Herzund Kreislaufforschung e.V. Published by Springer Medizin Verlag GmbH, ein Teil von Springer Nature - all rights reserved 2021

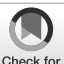

\section{Curriculum Kardiovaskuläre Intensiv- und Notfallmedizin (K-IN)}

\author{
Arbeitsgruppe und Task Force Kardiovaskuläre Intensiv- und \\ Notfallmedizin der Deutschen Gesellschaft für Kardiologie - \\ Herz- und Kreislaufforschung e. V., Düsseldorf (DGK)
}

Der Verlag veröffentlicht die Beiträge in der von den Autor*innen gewählten Genderform. Die Verwendung einer angemessenen gendergerechten Sprache, um Menschen in ihrer Vielfalt wertschätzend anzusprechen, wird begrüßt.

K. Werdan (Klinik und Poliklinik für Innere Medizin III,Universitätsklinikum Halle(Saale) der Martin-Luther-Universität Halle-Wittenberg, Halle (Saale), Deutschland), H.-R. Figulla (Jena, Deutschland), N. Frey (Universitätsklinikum Heidelberg, Heidelberg, Deutschland) waren als Beratertätig.

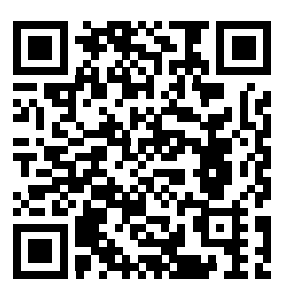

QR-Code scannen \& Beitrag online lesen

\section{Präambel}

Die Herz-Kreislauf-Medizin hat sich in allen ihren Facetten erheblich weiterentwickelt. Diese Entwicklung spiegelt sich auch in einer zunehmenden Spezialisierung innerhalb der Kardiologie wider.

Die Akademie für Aus-, Weiter- und Fortbildung der DGK trägt dieser Entwicklung Rechnung und hat unter Mitwirkung von Vertretern der jeweiligen Arbeitsgruppen Curricula für die Erlangung von Zusatzqualifikationen innerhalb der Kardiologie entwickelt. Diese Curricula sollen eine weitere Vertiefung des kardiologischen Wissens und technischer Fertigkeiten innerhalb des Fachgebiets erleichtern. Sie bauen also einerseits auf den Inhalten der (Muster-)Weiterbildungsordnung zum Facharzt für Innere Medizin und Kardiologie auf, gehen aber anderseits über das theoretische Wissen und die technischen und praktischen Fertigkeiten, die in der Weiterbildungsordnung für den Bereich Kardiologie gefordert werden, hinaus.

Für diese Curricula wurde der Begriff Zusatzqualifikation gewählt, um diese zusätzliche Qualifizierung unter dem
Dach der wissenschaftlichen Fachgesellschaft von der Regelweiterbildung nach der (Muster-)Weiterbildungsordnung der Bundesärztekammer zu unterscheiden. Im nachstehenden Text werden Personenbezeichnungen einheitlich und neutral für alle Geschlechter verwendet (also z.B. Kandidat für Kandidat und Kandidatin). Weiterhin werden der besseren Lesbarkeit halber nur die Facharztbezeichnungen der novellierten Weiterbildungsordnung (WBO) verwendet, sie beziehen in diesem Zusammenhang aber auch die entsprechenden Schwerpunktbezeichnungen der früheren WBO mit ein. 


\section{Inhaltsverzeichnis}

1. Einleitung

2. Ziele

3. Syllabus

4. Durchführung der Qualifizierung

5. Anforderungen an Kandidaten

6. Anforderungen an die Stätte der Zusatzqualifikation

7. Anforderungen an Leiter und stellv. Leiter der Zusatzqualifikation

8. Evaluierung

9. Anerkennung

10. Übergangsregelung

11. Rezertifizierung

12. Tabellarische Auflistung aller zu erfüllenden Voraussetzungen und einzureichenden Unterlagen

13. Qualitätskontrolle Literatur

\section{Abkürzungen}

ACS Acute coronary syndrome

AG3 Arbeitsgruppe Kardiovaskuläre Intensiv- und Notfallmedizin. Eine Arbeitsgruppe der DGK, welche die kardiovaskuläre Intensiv- und Notfallmedizin in der Kardiologie vertritt.

ARDS Acute respiratory distress syndrome

CAP Community acquired pneumonia

CPU Chest Pain Unit

DEGUM Deutsche Gesellschaft für Ultraschall in der Medizin e.V.

DGIIN Deutsche Gesellschaft für Internistische Intensiv- und Notfallmedizin e.V.

DGIM Deutsche Gesellschaft für Innere Medizin e.V.

DGK Deutsche Gesellschaft für Kardiologie Herz- und Kreislaufforschung e.V.

DIVI Deutsche Gesellschaft für Interdisziplinäre Intensivmedizin

ECLS Extracorporeal life support

ECMO Extrakorporale Membranoxygenierung

eCPR Extrakorporale kardiopulmonale Reanimation

HAP Hospital acquired pneumonia

HFU Heart Failure Unit

$I A B P$ Intraaortale Ballonpumpe

ICU Intensive Care Unit

IMC Intermediate Care Unit

LVAD Linksherzunterstützungssystem

MODS Multi organ dysfunction syndrome

NAST Notaufnahmestation

$\mathrm{PCl}$ Perkutane koronare Intervention

PiCCO Pulse contour cardiac output

STEMI ST-Strecken-Hebungsinfarkt

TAVI Transkatheteraortenklappenimplantation

TEE Transösophageale Echokardiographie

TTE Transthorakale Echokardiographie

Das Curriculum Kardiovaskuläre Intensiv- und Notfallmedizin beschreibt einen Qualifizierungsprozess für eine Zusatzqualifikation, welche über das Ausmaß der Weiterbildung zum Facharzt für Innere Medizin und Kardiologie und die Zusatzweiterbildung (Internistische) Intensivmedizin hinausgeht und diese um die für in den entsprechenden Bereichen tätigen Kardiologen notwendigen Kenntnisse erweitert. Während eines Zeitraums von kumulativ 24 Monaten - unter Einbeziehung der 18-monatigen Zusatzweiterbildung (ZWB) (Internistische) Intensivmedizin mit überwiegender Tätigkeit im Bereich der kardiovaskulären Intensivstation, Intermediate Care Station, Coronary Care Unit, Heart Failure Unit, Notaufnahme, Notaufnahmestation und Chest Pain Unit (CPU) eignet sich der Kandidat das erforderliche theoretische Wissen und die praktischen Fähigkeiten an, um selbstständig Patienten mit komplexen kardiovaskulären Erkrankungen während der intensiv- und notfallmedizinischen Phase zu behandeln. Die Kandidaten müssen als Voraussetzung für die Zusatzqualifikation über den Facharzt für Innere Medizin und Kardiologie verfügen. Weiterhin ist die Zusatzweiterbildung (Internistische) Intensivmedizin Voraussetzung für die Erteilung der Zusatzqualifikation Kardiovaskuläre Intensivund Notfallmedizin, die in 24 Monaten erlangt werden kann. Die Stätten, in denen die Zusatzqualifikation erworben werden kann, müssen über die erforderliche Infrastruktur (Klinik/eigenständige Abteilung für Kardiologie, zertifizierte Chest Pain Unit oder Heart Failure Unit [d.h. entweder HFU-Klinik oder HFU-Zentrum], kardiologisch (mit-)geführte Intensivstation [mind. 6 Beatmungsbetten]), 24-h-Dienst für Interventionelle Kardiologie, mindestens 2 Kardiologen mit Zusatzweiterbildung (Internistische) Intensivmedizin sowie über einen Leiter und einen stellvertretenden Leiter der Zusatzqualifikation (Supervisoren) verfügen. Die Zertifizierung der Kandidaten sowie der Stätten und ihrer (stellv.) Leiter erfolgt durch Gutachter der DGK. Eine Übergangsregelung für qualifizierte Kardiologen auf dem Gebiet der Kardiovaskulären Intensiv- und Notfallmedizin ist vorgesehen (vgl. Ziff. 10).

\section{Schlüsselwörter}

Kardiovaskuläre Intensiv- und Notfallmedizin · Internistische Intensivmedizin · Notfallmedizin . Chest Pain Unit · Interventionelle Kardiologie · Qualifizierung · Curriculum

\section{Einleitung}

Die kardiovaskuläre Intensiv- und Notfallmedizin ist eine Teildisziplin der Kardiologie, die sich seit der Einführung der Intensivstationen „Intensive Care Unit" (ICU), Intermediate-Care-Stationen (IMC), Heart Failure Units (HFU), Notaufnahme, Notaufnahmestationen (NAST) und Chest Pain Units (CPU) kontinuierlich weiterentwickelt hat und einen elementaren Bestandteil der Versorgung kardiovaskulärer Intensiv- und Notfallpatienten darstellt. Als Folge der Verbesserung der Techniken wurden interventionelle Verfahren zunehmend auf komplexere Befunde und multimorbide Patienten ausgedehnt. Die verbesserte Prognose der kardiovaskulären Patienten führt zu einer Steigerung der Fallzahlen, aber auch zu einer Zunahme von älteren Patienten sowie Patienten mit komplexen Begleiterkrankungen, wie z.B. Niereninsuffizienz oder Tumorerkrankungen oder auch solchen, die unter Immunsuppression stehen und so ein erhöhtes
Komplikationsrisiko haben. Um sowohl kardiovaskuläre Notfallpatienten als auch Patienten nach komplexen Eingriffen sicher zu versorgen, bedarf es daher einer spezialisierten kardiovaskulären Notfallund Intensivmedizin. Außerdem stellen neue kardiologische Verfahren, wie z.B. die kathetergestützte Klappentherapie, Herausforderungen an Diagnostik und Therapie, welche spezielle kardiologische Expertise auf Intensivstationen und in Notfalleinheiten erfordern.

Die kritisch Kranken auf der ICU/IMC/ CPU/HFU, die durch Kardiologen entweder bereits vorher behandelt wurden oder von anderen Abteilungen wegen akuter kardiovaskulärer Erkrankungen auf die ICU/IMC/CPU/HFU aufgenommen werden, erfordern hoch spezialisierte medizinische Leistungen, welche eine entsprechend große praktische und klinische Erfahrung erfordern. Daneben ist eine spezifische theoretische und praktische Expertise, aber auch Wissen über die kardiovaskuläre Pathophysiologie not- 
wendig. Moderne Akutmedizin erfordert aber auch Wissen aus anderen internistischen Bereichen/Fächern (z. B. Nierenersatzverfahren, Beatmungs-, Ernährungs-, Antibiotikatherapie). Der intensivmedizinisch tätige Kardiologe muss eine entsprechende Kompetenz erlangen und sich kontinuierlich auch in den anderen internistischen Bereichen weiterbilden. Auch bei der Akutversorgung von kardiovaskulären Patienten in der Notaufnahme, NAST und CPU ist kardiovaskuläre Kompetenz gefragt. Die rasche zeitgerechte Versorgung akuter kardiovaskulärer Erkrankungen kann dabei zur Senkung der Mortalität entscheidend beitragen.

In ca. 1250 Notaufnahmen, 323 zertifizierten CPUs und ca. 1350 konservativen Intensivstationen in ca. 1450 Krankenhäusern in Deutschland werden kardiovaskuläre Patienten internistisch, interdisziplinär und/oder kardiologisch versorgt. Zirka $60 \%$ dieser Patienten haben internistische Erkrankungen und von diesen wiederum ca. 2/3 kardiovaskuläre Erkrankungen (Survey DGIM). Somit stellt die kardiovaskuläre Versorgung von notfall- und intensivmedizinischen Patienten einen bedeutenden Anteil innerhalb der Kardiologie dar. Neue komplexe Verfahren wurden in der kardiovaskulären Intensivmedizin in den letzten Jahren etabliert (z. B. venoarterielle ECMO bzw. ECLS und weitere mechanische Herz-Kreislauf-Unterstützungssysteme), aber auch teilweise wieder weitgehend verlassen (IABP bei infarktbedingtem kardiogenem Schock). Ebenso sind weitere neue Verfahren zu erwarten, welche eine spezialisierte Ausbildung und eine enge Kooperation mit Nachbardisziplinen (Neurologie, Herzchirurgie, Anästhesie) erfordern.

Die kardiovaskuläre Intensiv- und Notfallmedizin verlangt vom durchführenden Arzt umfangreiche Kenntnisse und Erfahrungen auf dem gesamten Gebiet der Diagnostik und Therapie der Intensiv- und Notfallmedizin, welche nicht in vollem Umfang während der Weiterbildung zum Facharzt für Innere Medizin und Kardiologie bzw. der Zusatzweiterbildungen Internistische Intensivmedizin oder Notfallmedizin vermittelt werden können.

Zusätzliche kardiovaskuläre Ausbildungsinhalte werden für die Erlangung der Zusatzbezeichnung Kardiovaskuläre
Intensivmedizin bzw. Notfallmedizin vermittelt. Dies rechtfertigt den freiwilligen Erwerb vertiefender Kenntnisse, Erfahrungen und Fähigkeiten in der kardiovaskulären Intensiv- und Notfallmedizin, wobei im Sinne einer Qualitätssicherungsmaßnahme ein Curriculum angeboten werden soll, welches gemeinsam von der Arbeitsgruppe Kardiovaskuläre Intensiv- und Notfallmedizin der DGK sowie der Task Force kardiovaskuläre Intensiv- und Notfallmedizin der DGK ausgearbeitet wurde.

Folgende Leitlinien und Empfehlungen sind dem Curriculum zugrunde gelegt:

1. Leitlinien der Deutschen Gesellschaft für Interdisziplinäre Intensivmedizin (DIVI) zum „Betreiben einer Intensivstation" [1],

2. Positionspapier Internistische Intensivmedizin [5]

3. Acute Cardiac Care Curriculum and Intensive Cardiovascular Care Unit [2, 10]

4. Kriterien der DGK für "Chest Pain Unit", Update 2016 und Update 2020 [3, 6]

5. Empfehlungen Aufbau und Organisation von Herzinsuffizienz-Netzwerken (HF-NETs) und Herzinsuffizienz-Einheiten (Heart Failure Units) zur Optimierung der Behandlung der akuten und chronischen Herzinsuffizienz [7]

6. Empfehlungen zur Ultraschallausbildung in der Internistischen Intensivund Notfallmedizin: Positionspapier der DGIIN, DEGUM und DGK $[4,8,9]$.

\section{Ziele}

Ziel des Curriculums ist die Beschreibung eines Qualifizierungsprozesses für spezialisierte kardiovaskuläre intensivund notfallmedizinische Kompetenzen (Zusatzqualifikation) zur Versorgung kardiovaskulärer Notfälle und kritisch kranker kardiovaskulärer Patienten in Deutschland.

Damit soll die Qualifizierung in diesem spezialisierten Bereich über das Ausmaß der allgemeinen Facharztweiterbildung in der Kardiologie und die Zusatzweiterbildungen (Internistische) Intensivmedizin und Notfallmedizin sowie Klinische Akut- und Notfallmedizin hinaus erweitert werden.

Die Qualifizierung soll in einem definierten und von der Fachgesellschaft (DGK) zertifizierten Prozess durchgeführt werden, welcher eine Mindestqualität der Zusatzqualifikation sichern und dadurch nachhaltig die Versorgungsqualität kardiovaskulärer Notfall- und Intensivpatienten verbessern soll.

Die Anerkennung betrifft sowohl die Überprüfung der von den zu qualifizierenden Kandidaten geforderten Qualifikationen als auch die Zertifizierung von Stätten der Zusatzqualifikation.

\section{Syllabus}

\section{Qualifizierungsinhalte}

Folgende Inhalte sollen während der Qualifizierung in der kardiovaskulären Intensivund Notfallmedizin vermittelt werden:

Kardiologische Notfall-Diagnostik, -Monitoring und -Behandlung

- Notfallechokardiographie/-sonographie

- Hämodynamisches Monitoring und Management

- Management der akuten Herzinsuffizienz (inklusive des akuten Rechtsherzversagens)

- ACS- und STEMI-Versorgung in der Akutmedizin

- Management des infarktbedingten kardiogenen Schocks

- Mechanische Kreislaufunterstützungsverfahren

- Notfall-Rhythmusdiagnostik und akutes Arrhythmiemanagement

- Reanimation/ALS

- Leitsymptom Dyspnoe und Brustschmerz

- Hypertensive Notfälle

- Hypotonie und Synkope

- Herzerkrankung bei speziellen Patientengruppen (HIV, Schwangerschaft, Tumorpatienten, Patienten mit pulmonalarteriellem Druck, Tranplantationspatienten, akutes Rechtsherzversagen)

- Pharmakotherapie in der kardiovaskulären Notfallmedizin

- Notfälle bei Device-Patienten

Kardiologische intensivmedizinische Erkrankungen

- Lungenembolie inklusive pulmonale Hypertonie

- Dekompensierte Herzklappenfehler 
- Herzstillstand/kardiopulmonale Reanimation

- Postreanimationsphase und PostCardiac-Arrest-Syndrom

- Psychosomatische Aspekte von Herzkrankheiten unter dem Aspekt der Stresssituation einer Intensiv- oder Notfallstation

- Akutes Koronarsyndrom

- Kardiogener Schock

- Akutes Herzversagen inklusive akutes Rechtsherzversagen

- Kritische Rhythmusstörungen

- Hypertensive Notfälle

- Kardiomyopathien

- Akutes Aortensyndrom

- Thrombosen

- Akute Gefäßkrankheiten (Gefäßverschlüsse, akute Ischämien)

- Überwachung nach kardiologischen und herzchirurgischen Eingriffen

- Patienten nach Herztransplantation und LVAD-Patienten

\section{Diagnostik und Monitoring}

- Spez. TTE-Diagnostik

- Spez. TEE-Diagnostik

- Rechtsherzkatheteruntersuchung

- Linksherzkatheteruntersuchung

- Beurteilung komplexer EKGs

- Schrittmacher- und Device-Abfrage

- Biomarkerdiagnostik

- Fokussierter Gefäßultraschall

- Perikardpunktion

- Kardiale CT/MRT-Diagnostik

- Myokardbiopsie

Therapeutische Verfahren

- Akute PCl

- Perikardpunktion

- Passagere Schrittmacheranlage

- Differenzierte Inotropika-/Vasopressorentherapie

- Zielgerichtetes Temperaturmanagement (TTM) (milde therapeutische Hypothermie)

- Elektrotherapie - Kardioversion, Defibrillation, Überstimulation

- Mechanische Kreislaufunterstützung

- Notfallvalvuloplastie

- Thrombolyse

- Thrombektomie

- Thrombusfragmentierung
Notfallatemwegsmanagement

- Endotracheale Intubation und perkutane Dilatationstracheotomie/ Larynxtubus

- Ultraschalldiagnostik und Bronchoskopie zum Atemwegsmanagement

- Prinzipien der Beatmungstherapie einschließlich nichtinvasiver Beatmung

- Weaning und prolongiertes Weaning

Pneumologische Begleiterkrankungen bei kardiovaskulären

Patienten

- (Stauungs-)Pneumonie

- ARDS

- COPD/Asthma

Neurologische Begleiterkrankungen bei kardiovaskulären Patienten

- Leitsymptom Koma- und Bewusstseinsstörungen

- Ischämischer Schlaganfall

- Intrazerebrale Blutung

- Bakterielle Meningitis/Meningoenzephalitis

- Epilepsie/Status epilepticus

- ICU-acquired Weakness (ICUAW) (CIP, CIM, kognitive Dysfunktion)

- Management von Analgosedierung und Delir

- Irreversibler Hirnfunktionsausfall (Hirntoddiagnostik)

- Posttraumatisches Belastungssyndrom

Nephrologische Begleiterkrankungen bei kardiovaskulären

Patienten

- Leitsymptom Oligurie/Anurie

- Akutes Nierenversagen

- Nierenersatzverfahren

- Notfälle bei Dialysepatienten

Gastroenterologische Begleiterkrankungen bei kardiovaskulären Patienten

- Leitsymptom akutes Abdomen

- Akute gastrointestinale Blutung

- Clostridium-difficile-assoziierte Erkrankung

- Akutes Leberversagen

- Leberzirrhose und Komplikationen

- Ernährung (enteral und parenteral)

- Akute Pankreatitis

- Abdominelles Kompartmentsyndrom
Infektiologische Aspekte bei

kardiovaskulären Patienten

- Patienten mit geschwächtem Immunsystem

- Infektion, Prävention und mikrobiologische Diagnostik

- Fieber inklusive Fieber in Aplasie und Fieber unklarer Genese

- Sepsis, septischer Schock und MODS

- Pneumonie (CAP, HAP)

- Multiresistente Problemkeime in der Akutmedizin

Postoperative Überwachung und Bildgebung bei kardiovaskulären

Patienten

- Blutung und Thrombose

- Klinische Bildgebung (CT, MRT, Echo, Sonographie, Thoraxröntgenaufnahme)

- Hochrisikopatienten, präoperative Risikoevaluierung, postoperative Betreuung

- Multimorbide Patienten

- Postoperative- und postinterventionelle-Patienten ( $\mathrm{PCl} /$ Herzinfarktpatienten, TAVI-Patienten, ECMO-Patienten, PCIPatienten, koronare Bypasspatienten)

Datenerhebung in der Notaufnahme/Intensivstation

- Klinische Ergebnisse

- Mortalität in der Notaufnahme

- Mortalität in der CPU

- Mortalität auf der Intensivstation

- Beurteilung von technischen Innovationen

- Organisation und Management

- Intra- und interhospitale Transportbegleitung

- Qualitätssicherung und Wirtschaftlichkeit auf der Intensivstation

- Intensivmedizinische/notfallmedizinische Scores

Prozessqualität, beruflichprofessionelles Verhalten, Ethik und

Palliativmedizin

- Klinische Untersuchung

- Strukturierte Visite

- Kommunikation auf der Intensivstation und in der Notaufnahme (,shared decision making" des Stationsteams mit Patienten, Angehörigen und gesetzlichen Betreuern)

- Ethik in der Akutmedizin 
- Palliativmedizin in der Akutmedizin

- Organspende und Transplantation

- Angehörigengespräche

- Ethisches Fallgespräch

- End-of-Life-Entscheidungen

- Weiterbildung

- Fehlermanagement

- Teamführung

- Budgetführung/-gestaltung

\section{Qualifizierungsziele (überwiegend praktische Erfahrungen und Fertigkeiten)}

Im Detail sind für die Zusatzqualifikation in der kardiovaskulären Intensiv- und Notfallmedizin folgende Qualifizierungsziele definiert:

- Bildgebung in der Akutmedizin:

- fokussierte Echokardiographie (z. B. dekompensierte Vitien),

- fokussierte Abdomensonographie,

- fokussierte Lungen-/Thoraxsonographie,

- fokussierte transösophageale Echokardiographie (Beurteilung dekompensierte Vitien),

- Intubation und supraglottische Atemwegshilfen,

- Anlage von Gefäßzugängen (inklusive ultraschallgesteuerter Punktionstechnik),

- differenzierte EKG-Diagnostik,

- Einsatz von und Diagnostik mit unterschiedlichen Monitoringsystemen,

- differenzierte Inotropika-/Vasopressorentherapie,

- medikamentöse Modulation der Vorund Nachlast,

- Pleura-, Perikard-, Lumbal- und Aszitespunktion,

- Thoraxdrainage,

- Punktionstracheotomie,

- Nierenersatzverfahren und differenzierte Therapie,

- Kontrolle/Programmierung von Defibrillator/Schrittmacher,

- Kardioversion/Defibrillation,

- transvenöser temporärer Schrittmacher,

- invasive Beatmung, nichtinvasive Beatmung (NIV) und high-flow-nasale Oxygenierung,

- flexible Bronchoskopie,
- Aufbau und Anlage einer venovenösen/venoarteriellen ECMO,

- Aufbau anderer mechanischer HerzKreislauf-Unterstützungssysteme (z. B. links-/rechtsventrikuläre Mikroaxialpumpe oder Tandem-Heart),

- eCPR,

- Punktion Perikarderguss.

Zur Qualitätskontrolle muss der Supervisor den Qualifizierungsprozess zu gegebener Zeit im Zeugnis beurteilen, ob der Kandidat ausreichend Erfahrung und Fertigkeiten besitzt bzw. erworben hat, um diese Prozeduren in Eigenverantwortung durchzuführen.

\section{Durchführung der Qualifizierung}

\section{Dauer}

Die zusätzliche Qualifizierung gemäß Curriculum Kardiovaskuläre Intensiv- und Notfallmedizin dauert in Summe 24 Monate; 18 Monate können aus der Zeit zur Erlangung der Zusatzweiterbildung (Internistische) Intensivmedizin angerechnet werden, wenn entsprechende Qualifikationen nachgewiesen werden. Für die Anerkennung der Qualifikationen werden die Zusatzweiterbildung Intensivmedizin und der Facharzt Innere Medizin und Kardiologie (bestandene Prüfungen, Zeugnis) vorausgesetzt.

Die Tätigkeit hat überwiegend im Bereich der kardiovaskulären Intensiv- und Notfallmedizin stattzufinden. Die Qualifizierung sollte über Blöcke von jeweils 3 bis 12 Monaten durch eine vollzeitige Einteilung auf der internistischen/ kardiovaskulären Intensivstation/HFU und/oder Notaufnahme/CPU stattfinden.

Die Qualifizierung kann auch in mehreren Abschnitten durchgeführt werden, wobei jeder Abschnitt mindestens 3 Monate betragen sollte.

Die formalen Lernprozesse werden von Gutachtern der DGK überprüft (Logbuch und Dokumentation) und zertifiziert.

Die Qualifizierung wird an DGK-zertifizierten Stätten der Zusatzqualifikation (s. Ziff. 6) durchgeführt. Der Leiter der Zusatzqualifikation muss sicherstellen, dass die Kandidaten die notwendigen theoretischen Kenntnisse („,knowledge ${ }^{\prime \prime}$ ) und die praktischen Fähigkeiten („,skills“) über alle Teilbereiche des Syllabus erwerben.

Hierzu gehören:

a) Lerneinheiten und Kurse

- Die Kandidaten müssen an akkreditierten formalen Lerneinheiten, die national oder im Ausland organisiert werden und ihren fachlichen Schwerpunkt im Bereich der kardialen Notfall- und Intensivmedizin haben, teilnehmen (DGK, DGIIN, DIVI, ACCA, ESICM) und die Teilnahmebescheinigungen nachweisen.

- Formales Lernen schließt die Teilnahme an nationalen und internationalen Kursen und Kongressen im Bereich der kardiovaskulären Intensiv- und Notfallmedizin ein (z.B. nationale Fortbildungen und Kurse der DGK [z. B. CPU-Kompakt, ACLS-Kurs, Fit for ICU] oder Kurse vergleichbaren Inhaltes anderer Anbieter).

- Als Fortbildung werden auch Simulationskurse empfohlen; zum Erlernen praktisch-manueller Fähigkeiten außerhalb der Patientenbehandlung auf der Intensivstation (z. B. DGKkardiovaskuläre Intensiv- und Notfallmedizin, DGK-Kurs zur klinischen Akut- und Notfallmedizin).

- Zur Qualifizierung müssen insgesamt Weiterbildungen/Kurse von mindestens 25 CME-Punkten/Jahr (insgesamt mindestens 50 CMEPunkte) nachgewiesen werden.

- Die Teilnahme an lokalen, zertifizierten Fortbildungen in der Stätte der Zusatzqualifikation (z. B. JournalClubs, M-und-M-Konferenzen) wird als Fortbildung anerkannt.

- Das Literaturstudium (Fachzeitschriften, Lehrbücher, Internet) wird als Teil des Qualifizierungsprozesses vorausgesetzt.

b) Dokumentation der Untersuchungen und Fallbesprechungen (Logbuch)

- Die im Rahmen der Qualifizierung vom Kandidaten durchgeführten Behandlungen und Maßnahmen im Bereich der kardiovaskulären Intensiv- und Notfallmedizin sind in einem von der DGK zur Verfügung gestellten Logbuch zu dokumentieren. 


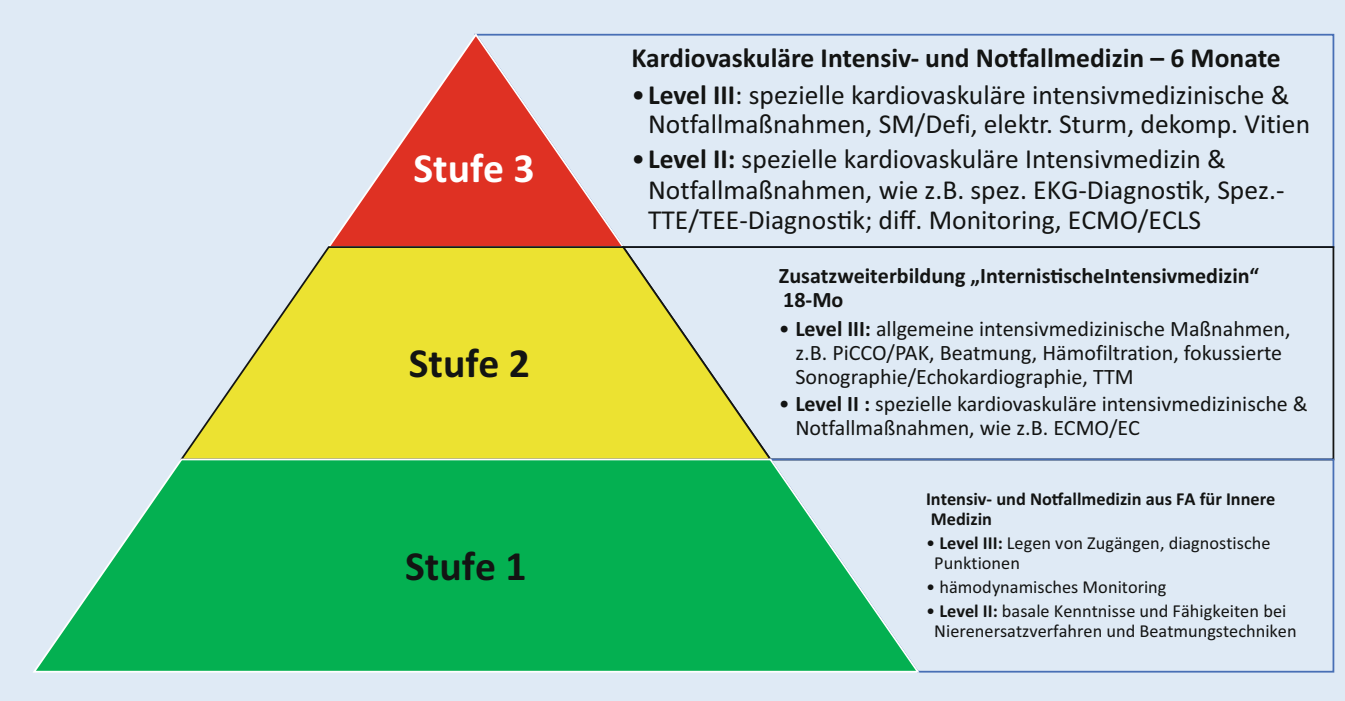

Abb. 1 Curriculum Kardiovaskuläre Intensiv- und Notfallmedizin mit verschieden Stufen. SM Schrittmacher, Defi Defibrillator, TTE transthorakales Echo, TEE transösophageales Echo, ECMO extrakorporale Membranoxygenierung, $E C L S_{\text {"extracirculatory }}$ life support", PAC pulmonalarterieller Katheter, TTM "targeted temperature management")

- Eine regelmäßige Teilnahme an fallorientierten Konferenzen (z. B. M-und-M-Konferenzen, CPR-Nachbesprechungen, Ethik-Runden/-Fallkonferenzen) wird erwartet; interdisziplinäre Zusammenarbeit und Teilnahme an chirurgischen und/ oder anästhesiologischen Konferenzen mit Schwerpunkt Intensiv- und Notfallmedizin werden gewünscht.

c) Wissenschaft

Allen Kandidaten wird innerhalb ihres Qualifizierungsprogramms empfohlen, an wissenschaftlichen Projekten auf dem Gebiet der kardiovaskulären Intensiv- und Notfallmedizin mitzuarbeiten.

Des Weiteren wären wünschenswert:

- Teilnahme an Studien im Bereich der kardiovaskulären Intensiv- und Notfallmedizin, Teilnahme an und/ oder Präsentationen auf Kongressen,

- Teilnahme an kardiovaskulären Qualitätsinitiativen der DGK (z. B. FITTSTEMI-Programm, CPU-Register, nationales kardiogenes SchockRegister etc.).

\section{Modulkurse Kardiovaskuläre} Intensiv- und Notfallmedizin der DGK

Im Qualifizierungszeitraum müssen insgesamt Weiterbildungen/Kurse von mindestens 25 CME-Punkten/Jahr (insgesamt mindestens 50 CME-Punkte) nachgewiesen werden. Diese können z. B. bei folgenden Kursen erlangt werden:
- DGK-Fit for ICU,

- DGK-CPU Kompakt,

- DGK-ACLS-Kurs,

- DGK-DIVI-ECMO-Kurs,

- DGK-Herzinsuffizienz Grund- und Aufbaukurs.

In Vorbereitung ist der folgende Kurs:

- DGK-Kurs - Kardiovaskuläre Intensivund Notfallmedizin

- Akute Herzinsuffizienz und Schock

- ACS- und Infarktversorgung

- Behandlung von dekompensierten Vitien

- Organersatz (Niere, Leber)

- Hämodynamik und Monitoring

- EKG

- Pharmakotherapie auf der ITS

- Struktur und Funktion auf der ITS (Struktur, Ethik und Ökonomie)

- SM- und Device-Therapie

- Mechanische Kreislaufunterstützung

- Akuter und chronischer Lungenhochdruck

- Kardiorenales, kardiohepatisches, kardiopulmonales Syndrom

- Therapie des Schlaganfalls

Alternativ können auch Kurse anderer Veranstalter (z.B. DGIIN, DIVI, ACVC, ESICM) mit vergleichbaren Inhalten anerkannt werden.

\section{Ablauf der Qualifizierung}

Die Absolvierung der Qualifizierung erfordert einen Zeitraum von insgesamt 24 Monaten, wobei bei Unterbrechungen die ein- zelnen Module/Rotationen 3 Monate nicht unterschreiten dürfen. Es gibt 3 Stufen, die aus dem Intensivteil der Facharztausbildung, der Zusatzsatzqualifikation Internistische Intensivmedizin und dem Teil der kardiovaskulären Intensiv- und Notfallmedizin bestehen.

Die Dauer der 3. Stufe kann jedoch individuell bis zur maximalen Qualifizierungsdauer verlängert werden, sofern der Supervisor (Definition s. Ziff. 7) die erforderlichen individuellen Fortschritte und Kenntnisse nicht feststellt. Der tatsächliche Wechsel des Kandidaten in die nächste Stufe wird vom Supervisor festgelegt, abhängig von den individuellen Fortschritten und Kenntnissen des Kandidaten.

Schwerpunkt der Tätigkeit des Kandidaten während der Zusatzqualifikation ist die ICU/IMC/HFU, Notaufnahme/NAST und CPU.

Lernen (theoretische Kenntnisse und praktische Fähigkeiten) unter Aufsicht ist der wichtigste Teil des Qualifizierungsprozesses im Bereich der kardiovaskulären Intensiv- und Notfallmedizin. Die Kandidaten sollen die Diagnostik und Behandlung in der kardiovaskulären Akutmedizin anhand von etablierten Protokollen und Teamentscheidungen mit einem Supervisor diskutieren können, welcher für eine hinreichende Betreuung bei der Tätigkeit auf der Intensivstation verantwortlich und als Leiter der Zusatzqualifikation von der DGK anerkannt ist. Sie sollen praktische Fähigkeiten erlernen und eine besondere Expertise in Bezug auf kardiovaskuläre 
Tab. 1 Mindestzahlen im Rahmen der Zusatzqualifikation Kardiovaskuläre Intensiv- und Notfallmedizin

\begin{tabular}{|l|l|}
\hline Anforderungen (Teil 2 und 3) & 100 \\
\hline Spezielle EKG-Befundung bei Intensiv-/Notfallpatienten & 100 \\
\hline Management akutes Koronarsyndrom, Biomarker und Imaging & 200 \\
\hline Echo für kardiovaskuläre Notfälle & 50 \\
\hline Behandlung kardiogener Schock & 100 \\
\hline Akute Herzinsuffizienz inklusive akutes Rechtsherzversagen & 200 \\
\hline $\begin{array}{l}\text { Rhythmusstörungen aus dem kardiovaskulären intensiv- und notfallmedizinischen Be- } \\
\text { Synkope }\end{array}$ & 30 \\
\hline $\begin{array}{l}\text { Herzstillstand/kardiopulmonale Reanimation inklusive Behandlung Post-Cardiac-Arrest- } \\
\text { Syndrom }\end{array}$ & 30 Breitkomplextachykardien, \\
\hline Perikarditis, Myokarditis, Endokarditis & 10 \\
\hline Hypertensive Notfälle & 30 \\
\hline $\begin{array}{l}\text { Herzerkrankung bei speziellen Patientengruppen (HIV, Schwangerschaft, Tumorpatien- } \\
\text { ten, Patienten mit PAH) }\end{array}$ & 20 \\
\hline Behandlung von Transplantationspatienten inklusive Auswahl Organspende & 10 \\
\hline Behandlung von Device-Patienten & 20 \\
\hline Fehlermanagement/M-und-M-Konferenzen & 20 \\
\hline Mechanische Organersatzpatienten (z. B. Kunstherz) & 5 \\
\hline Hämodynamisches Monitoring (mindestens 2 Verfahren - z. B. PiCCO, Thermodilatation) & 150 \\
\hline
\end{tabular}

Patienten, Erkrankungen, Techniken und Methoden erlangen.

Das Curriculum Kardiovaskuläre Intensiv- und Notfallmedizin wird in 3 Stufen eingeteilt (- Abb. 1).

Stufe 1. Die Kandidaten sammeln Erfahrung und erlernen Basiskenntnisse in der kardiovaskulären Intensiv- und Notfallmedizin im Rahmen ihrer Weiterbildung zum Facharzt für Innere Medizin und Kardiologie. Hierzu gehören das Erlernen des Legens von Zugängen, von diagnostischen Punktionen, des hämodynamischen Monitorings und die Assistenz bei den Nierenersatzverfahren und Beatmungstechniken, die durch erfahrene Intensivmediziner angeleitet werden (6 Monate Intensiv- und Notfallmedizin des FA Innere Medizin und Kardiologie).

Stufe 2. Die Kandidaten erlernen neben der kardiovaskulären Intensiv- und Notfallmedizin die allgemeine Intensivmedizin unter enger Supervision im Rahmen der Erlangung der Zusatzweiterbildung (Internistische) Intensivmedizin. Sie assistieren bei komplexen Maßnahmen (z.B. Anlegen einer ECMO). Für die Planung der Behandlung von kardiovaskulären Intensivund Notfallmedizinpatienten und Unterstützung im Fall von Komplikationen steht

\section{Mindestzahlen}

Als Mindestzahlen gelten die Zahlen zur Erlangung der Zusatzweiterbildung Internistische Intensivmedizin (Stufe 1) und Zahlen des Schwerpunktes Internistische Intensivmedizin und Kardiovaskuläre Intensiv- und Notfallmedizin (Stufe 2 und 3; - Tab. 1).

\section{Anforderungen an den Kandidaten}

Eine grundlegende Ausbildung in Innerer Medizin und Kardiologie muss vor der Qualifizierung in der kardiovaskulären Intensiv- und Notfallmedizin vorhanden sein. Der Kandidat hat Erfahrungen in der Betreuung von Patienten mit internistischen und komplexen kardiologischen Erkrankungen im Stationsdienst nachzuweisen (inklusive Verordnung der medikamentösen Therapie, Anordnung der notwendigen nichtinvasiven Untersuchungsmethoden und Aufklärung für invasive Prozeduren; Bescheinigung durch Ausbilder).

Vor der Tätigkeit auf der Intensivstation muss die Ausbildung zur "Fachkunde zum Strahlenschutz nach der Röntgenverordnung" zumindest begonnen worden sein. Das Curriculum Kardiovaskuläre Intensivund Notfallmedizin kann schon während der Facharztweiterbildung zum Kardiologen begonnen werden. Entsprechende Zeiten werden anerkannt. Die Zusatzqualifikation Kardiovaskuläre Intensivund Notfallmedizin kann jedoch erst nach der Erlangung der Facharzt- bzw. Schwerpunktanerkennung Kardiologie und der Zusatzbezeichnung Internistische Intensivmedizin abgeschlossen und erteilt werden.

Kandidaten aus dem nichteuropäischen Ausland, die sich um eine Stelle im Rahmen eines kardiovaskulären intensivund notfallmedizinischen Trainingsprogramms in Deutschland bewerben, müssen eine Ausbildung in Innerer Medizin und Kardiologie bzw. Internistischer Intensivmedizin nachweisen, welche von einer deutschen Landesärztekammer für eine ärztliche Berufsausübung in Deutschland akzeptiert wird. 


\section{Anforderungen an die Stätte der Zusatzqualifikation}

Folgende Anforderungen werden an die Stätte der Zusatzqualifikation gestellt:

- Leitung der Intensivstation bzw. CPU und/oder Notaufnahme durch einen Kardiologen, alternativ Behandlungshoheit für die kardiovaskuläre Patientenversorgung durch den Kardiologen,

- Leitung der curricularen kardiovaskulären intensiv- und notfallmedizinischen Qualifizierung durch einen Kardiologen (mit persönlicher Anerkennung der Zusatzqualifikation Kardiovaskuläre Intensiv- und Notfallmedizin),

- Weiterbildungsstätte für die Zusatzbezeichnung Internistische Intensivmedizin.

Die Stätten der Zusatzqualifikation müssen eine ICU/IMC/HFU/CPU mit kardiovaskulärem Schwerpunkt vorhalten, die dem Kandidaten die Möglichkeit geben, den Patienten vom Anfang bis zum Ende der Behandlung zu betreuen.

- Eine möglichst elektronische Dokumentation der diagnostischen und therapeutischen Maßnahmen der Intensivstation inklusive Schweregrad der Erkrankungen, Pflegeaufwand etc. soll von den zuständigen Gutachtern der DGK überprüft werden, um sicherzustellen, dass Maßnahmen in adäquater Zahl und Art als Voraussetzung für das Qualifizierungsprogramm durchgeführt werden können.

\section{Notaufnahme/-station/CPU}

- Die Stätten der Zusatzqualifikation sollen eine Notaufnahme/NAST mit kardiovaskulärem Schwerpunkt oder eine zertifizierte CPU vorhalten, die dem Kandidaten die Möglichkeit geben, den Patienten vom Anfang bis zum Ende der Notfallbehandlung zu betreuen.

- Eine Dokumentation der diagnostischen und therapeutischen Maßnahmen der Notaufnahme inklusive Art und Anzahl der Patienten, Pflegestandards, SOPs etc. soll von dem zuständigen Gremium der DGK überprüft werden, um sicherzustellen, dass Patienten in adäquater Zahl und Art als
Voraussetzung für das Qualifizierungsprogramm vorhanden sind.

\section{Anzahl durchgeführter Maßnahmen} und Behandlungen

- Eine kardiovaskuläre intensiv- und notfallmedizinische Qualifizierungsstätte sollte ein für die Qualifizierung ausreichendes Kontingent und Spektrum an Notfall- und Intensivpatienten, insbesondere kardiovaskulärer intensiv- und notfallmedizinischer Prozeduren und Maßnahmen aufweisen. Das Zentrum muss sicherstellen, dass der Kandidat die im Curriculum geforderten Untersuchungen (s. oben „Mindestzahlen“) durchführen kann.

- Ausstattungsmerkmale: Notaufnahme/NAST/CPU: mindestens 4 Patientenbetten Intensiv- und IMC-Station: mindestens 10 Betten oder 1000 Patienten pro Jahr mindestens 6 Beatmungsbetten, Nierenersatzverfahren, Herz-Kreislauf-Unterstützungssysteme (ggf. in Kooperation mit Herzchirurgie), Rechtsherzkatheter und Thermodilutionsverfahren

- Die von der DGK für die Anerkennung benannten Gutachter entscheiden über die Qualifikation der Klinik als Stätte der Zusatzqualifikation Kardiovaskuläre Intensiv- und Notfallmedizin. Neben der Art und Anzahl der kardiovaskulären intensiv- und notfallmedizinischen Patienten sollen die Infrastruktur (z. B. Konferenzen, Verfügbarkeit von Bildgebungsverfahren etc.) und das Angebot an weiteren - auch optionalen - Qualifizierungsinhalten berücksichtigt werden (Teambildung, interprofessionelle Kommunikation, DRGs etc.).

Die Anerkennung als Stätte der Zusatzqualifikation ist befristet. Für eine Fortführung der Anerkennung bedarf es eines Antrags auf Rezertifizierung.

\section{Anforderungen an Leiter und stellv. Leiter der Zusatz- qualifikation}

Folgende Anforderungen gelten für den Leiter und den stellv. Leiter (Supervisoren) der Zusatzqualifikation:

- Zwei Supervisoren müssen verfügbar sein. Diese verfügen jeweils über eine Erfahrung von mindestens 2 Jahren in kardiovaskulärer Intensiv- und Notfallmedizin und sind im Besitz der Facharzturkunde Innere Medizin und Kardiologie, der Zusatzweiterbildung (Internistische) Intensivmedizin sowie der persönlichen Anerkennung der Zusatzqualifikation Kardiovaskuläre Intensiv- und Notfallmedizin.

- Leiter und stellv. Leiter der Zusatzqualifikation sollten überwiegend in der kardiovaskulären Intensiv- und Notfallmedizineinheit tätig sein.

- Leiter und stellv. Leiter der Zusatzqualifikation sind verantwortlich für die kardiovaskuläre Intensiv- und Notfallmedizineinheit und stellen sicher, dass die Kandidaten die notwendige Supervision erhalten, um die diagnostischen und therapeutischen Prozeduren entsprechend dem Curriculum zu erlernen.

- Außerdem stellen beide sicher, dass die Kandidaten an den formalen Lerneinheiten und Kursen teilnehmen sowie in die Ausbildungs- und Forschungsaktivitäten der Abteilung eingebunden sind.

- Leiter und stellv. Leiter der Zusatzqualifikation müssen in Vollzeit am Klinikum tätig sein.

\section{Evaluierung}

Die Evaluierung setzt sich aus folgenden Komponenten zusammen:

- Bericht des (stellv.) Leiters der Zusatzqualifikation

Dieser muss Details zu den Aktivitäten, der Kompetenz und der erreichten Selbstständigkeit des Kandidaten beinhalten. Er beinhaltet also neben Informationen zu den Kenntnissen und Erfahrungen auch eine Beschreibung der Fortschritte in praktischen Tätigkeiten und theoretischem Wissen. Es muss erkennbar sein, dass der Kandidat 
eigenverantwortlich kardiovaskuläre intensiv- und notfallmedizinische Behandlungen verantwortungsbewusst und sach- bzw. leitliniengerecht durchführen kann. Insbesondere sollen das Erkennen kritischer Befunde, das Management von schwierigen Fällen und die Interaktion mit dem Team, dem Patienten, Angehörigen und ggf. Betreuer beschrieben werden.

- Beleg der kontinuierlichen Fortbildung in der Subdisziplin durch Nachweis der Teilnahme an akkreditierten Kongressen, Workshops, Symposien und Kursen der Fachgesellschaften oder deren Mitglieder. Mindestens die Hälfte der Pflichtpunkte für ärztliche Fortbildung (25 Punkte pro Jahr) müssen im thematischen Schwerpunkt des Curriculums nachgewiesen werden [s. Ziff. 4. a)]).

- Dokumentation der durchgeführten Behandlungen und Maßnahmen in einem von der DGK zur Verfügung gestellten Logbuch. Sämtliche Prozeduren und die verantwortliche Stellung (verantwortlicher Ausführender oder Assistenz) müssen dokumentiert werden. Die Korrektheit des Logbuchs wird schriftlich durch den (stellv.) Leiter der Zusatzqualifikation bestätigt.

\section{Anerkennung}

Die Anerkennung der Klinik als Stätte der Zusatzqualifikation und des Leiters bzw. stellv. Leiters der Zusatzqualifikation erfolgt durch Gutachter der DGK. Die Anerkennung der Stätte wird durch den Kardiologen beantragt, welcher die Leitung der Zusatzqualifikation übernehmen wird. Die Gutachter sichten die Unterlagen und gewähren die Anerkennung, sofern die Voraussetzungen erfüllt sind.

Anträge der Kandidaten auf Zertifizierung der curricularen Leistungen zur Erlangung der Zusatzqualifikation werden von dem oben genannten Gremium geprüft. Vorzulegen sind:

- aktueller Lebenslauf,

- Facharztzeugnis für Innere Medizin und Kardiologie,

- Zeugnis über die Zusatzweiterbildung (Internistische) Intensivmedizin,

- Logbuch,
- Teilnahmebescheinigungen von Fortbildungsveranstaltungen/Kongressen etc. gemäß Ziff. 4. a) unter Angabe der mindestens einzureichenden CMEPunkte pro Qualifizierungsjahr,

- Teilnahmebescheinigungen von Kursen gemäß Ziff. 4. a) unter Angabe der genauen Kursbezeichnung,

- Beurteilung/Zeugnis des (stellv.) Leiters der Zusatzqualifikation.

Die DGK stellt dem Kandidaten ein Zertifikat über die Anerkennung der Zusatzqualifikation Kardiovaskuläre Intensivund Notfallmedizin aus, wenn alle Voraussetzungen hierfür erfüllt sind. Die Anerkennung ist befristet. Für eine Fortführung der Anerkennung bedarf es eines Antrags auf Rezertifizierung.

\section{0. Übergangsregelung}

Fachärzte für Innere Medizin und Kardiologie bzw. Innere Medizin mit Schwerpunkt Kardiologie, die nachweislich in mindestens 2 der letzten 5 Jahre vor Antragstellung überwiegend auf dem Gebiet der kardiovaskulären Intensiv- und Notfallmedizin tätig waren (unter Erfüllung der im Curriculum geforderten Mindestzahlen) und die Zusatzweiterbildung (Internistische) Intensivmedizin besitzen, können auf Antrag im Rahmen einer Übergangsregelung ohne formale Absolvierung des Qualifizierungsprogramms die Anerkennung der Zusatzqualifikation Kardiovaskuläre Intensiv- und Notfallmedizin erhalten.

Die überwiegende Tätigkeit auf dem Gebiet der kardiovaskulären Intensiv- und Notfallmedizin sowie die geforderten Mindestzahlen sind glaubhaft nachzuweisen, z. B. durch Bestätigung des Leiters der Einrichtung.

Die Anerkennung im Rahmen der Übergangsregelung ist befristet. Im Anschluss ist ggf. ein Antrag auf Rezertifizierung zu stellen.

Die Übergangsregelung gilt für 2 Jahre ab Publikation dieses Curriculums. Nach Ablauf dieser Frist ist die Erlangung der $\mathrm{Zu}$ satzqualifikation Kardiovaskuläre Intensiv- und Notfallmedizin nur noch durch Teilnahme an dem entsprechenden Qualifizierungsprogramm möglich.

\section{Rezertifizierung}

Vor Ablauf der Gültigkeit des jeweiligen Zertifikats muss eine Rezertifizierung erfolgen.

\section{Tabellarische Auflistung aller zu erfüllenden Voraussetzungen und einzureichenden Unterlagen}

Die für die Zertifizierung sowohl von Personen als auch von Stätten zu erfüllenden Voraussetzungen sind den dem Curriculum zughörigen Tabellen zu entnehmen.

\section{Qualitätskontrolle}

Die Stätten stellen dem Gremium auf Aufforderung die Dokumente der gesetzlich erforderlichen Qualitätskontrollen zur Verfügung.

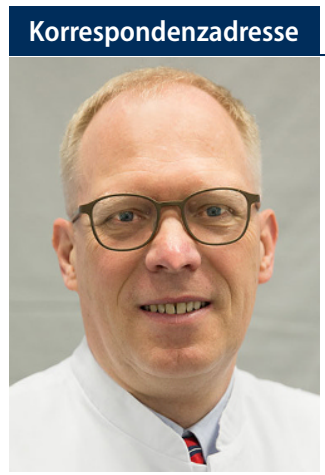

Prof. Dr. M. Buerke

Medizinische Klinik II, St. Marien-Krankenhaus Siegen

Kampenstr. 51, 57072 Siegen, Deutschland m.buerke@mariengesellschaft.de

\section{Einhaltung ethischer Richtlinien}

Interessenkonflikt. Den Interessenkonflikt der Autoren finden Sie online auf der DGK-Homepage unter http://leitlinien.dgk.org/ bei der entsprechenden Publikation.

Für diesen Beitrag wurden von den Autoren keine Studien an Menschen oder Tieren durchgeführt. Für die aufgeführten Studien gelten die jeweils dort angegebenen ethischen Richtlinien.

\section{Literatur}

1. Jorch G, Kluge S, König F, Markewitz K, Notz K, Parvu V, Quintel M, Schneider D, Sybrecht GW, Waydhas C (2010) Empfehlungen zur Struktur und Ausstattung von Intensivstationen. https:// www.divi.de/empfehlungen/publikationen/ 
intensivmedizin/399-empfehlungen-zurstruktur-von-intensivstationen-langversion/

2. Bonnefoy-Cudraz $E$, Bueno $H$, Casella G, De Maria E, Fitzsimons D, Halvorsen S, Hassager C, lakobishvili Z, Magdy A, Marandi T, Mimoso J, Parkhomenko A, Price S, Rokyta R, Roubille F, Serpytis P, Shimony A, Stepinska J, Tint D, Trendafilova $E$, Tubaro $M$, Vrints $C$, Walker $D$, Zahger D, Zima E, Zukermann R, Lettino M (2017) Acute Cardiovascular Care Association Position Paper on Intensive Cardiovascular Care Units: An update on their definition, structure, organisation and function. European Heart Journal: Acute Cardiovascular Care 7(1):204887261772426

3. Post F, Gori T, Giannitsis E, Darius $H$, Baldus $S$, Hamm C, Hambrecht R, Hofmeister HM, Katus $\mathrm{H}$, Perings S, Senges J, Münzel T (2015) Criteria of the German Society of Cardiology for the establishment of chest pain units: update 2014. Clin Res Cardiol 104:918-928

4. Michels G, Zinke H, Möckel M et al (2017) Empfehlungen zur Ultraschallausbildung in der internistischen Intensiv- und Notfallmedizin: Positionspapier der DGIIN, DEGUM und DGK. Kardiologe 11:285-290

5. Riessen R, Janssens U, Buerke M, Kluge S (2016) Position paper of the German Society for Medical Intensive Care Medicine and Emergency Medicine (DGIIN) on medical intensive care medicine. Med Klin Intensivmed Notfmed 111(4):295-301

6. Evangelos Giannitsis E, Post F, Haerer W, Bauersachs J, Thiele H, Buerke M, Mudra H, Möllmann H, Münzel T (2020) Kriterien der Deutschen Gesellschaft für Kardiologie - Herz- und Kreislaufforschung für "Chest Pain Units" Update 2020 Criteria of the German Cardiac Society for chest pain units Update 2020. Kardiologe 14:466-479. https://doi. org/10.1007/s12181-020-00417-w

7. ErtI G, Angermann CE, Bekeredjian R, Beyersdorf F, Güder G, Gummert J, Katus HA, Kindermann I, Pauschinger M, Perings S, Raake PWJ, Störk S, von Scheidt W, Welz S, Böhm M (2016) Aufbau und Organisation von Herzinsuffizienz-Netzwerken (HF-NETs) und Herzinsuffizienz-Einheiten („Heart Failure Units", HFUs) zur Optimierung der Behandlung der akuten und chronischen Herzinsuffizienz. Kardiologe 10:222 (Gemeinsame Empfehlungen der DGK und der DGTHG zur Behandlung der Herzinsuffizienz)

8. Michels G, Zinke H, Möckel M, Hempel D, Busche C, Janssens U, Kluge S, Riessen R, Buerke M, Kelm M, von Bardeleben RS, Knebel F, Busch H-J (2017) Recommendations for education in ultrasound in medical intensive care and emergency medicine: position paper ofDGIIN,DEGUM and DGK. Med Klin Intensivmed Notfmed 112(4):314-319

9. Hagendorff A, Fehske W, Flachskampf FA et al (2020) Manual zur Indikation und Durchführung der Echokardiographie - Update 2020 der Deutschen Gesellschaft für Kardiologie. Kardiologe 14:396-431

10. EBSC Subspecialty Curriculum Template Curriculum for acute cardiac care subspecialty training in europe (noyear available)

\section{Curriculum cardiovascular intensive care and emergency medicine (K-} IN). Working group and task force for cardiovascular intensive and emergency medicine of the German Cardiac Society

The curriculum cardiovascular intensive care and emergency medicine describes a training process to acquire an additional qualification, which exceeds the basic training in intensive care and emergency medicine essential for general and invasive cardiology board certification. During a cumulative period of 24 months, including the 18-month training in intensive care medicine, predominantly with activity in the cardiovascular intensive care unit, intermediate care station, coronary care unit, heart failure unit, emergency room, and chest pain unit (CPU), the candidate acquires the theoretical knowledge and practical skills required to independently treat patients with complex cardiovascular diseases during the intensive and emergency medical phase. As a prerequisite for the additional qualification, the candidates must be a specialist in internal medicine and cardiology. Furthermore, the additional training (internal) in intensive care medicine is a prerequisite for the acquisition of the additional qualification in cardiovascular intensive and emergency medicine. The additional qualification in cardiovascular intensive care and emergency medicine from the German Cardiac Society (DGK) can be obtained in 24 months. The facilities in which the additional qualification can be acquired must have the necessary infrastructure (clinic/independent department for cardiology, certified chest pain or heart failure unit, cardiologically managed intensive care unit, with at least 6 ventilation beds, 24-h service for interventional cardiology, at least 2 cardiologists with additional training (internal) in intensive care medicine as well as a head and a deputy head with the additional qualification (supervisors). The site is recognized and the candidate is certified by a committee of the DGK. A transitional arrangement for qualified cardiologists in the field of cardiovascular intensive care and emergency medicine is planned.

\section{Keywords}

Cardiovascular intensive care and emergency medicine · Intensive care medicine · Emergency medicine · Chest pain unit · Interventional cardiology · Qualification · Curriculum 